\title{
Problem difficulty as a function of relative frequency of correct responses
}

\section{Abstract}

The attempt was made to identify variables determining the order in which problem solving responses are made. On the initial problem, difficulty was inversely related to the relative frequency of the correct response. Over a series of problems, performance was more complexly related to relative frequency of the correct response, with the difficulty of identifying various relative frequency positions the additional factor.

\section{Problem}

Problem solving behavior may be characterized as a sequence of responses that is terminated by the occurrence of the correct response. The spew hypothesis (Underwood \& Schulz, 1960) states that the order of emission of verbal units is directly related to their frequency of experience. Applied to problem solving, the prediction is that initial attempts at solution will consist of high frequency responses, with responses of lower frequency attempted later. Difficulty of solution should thus be inversely related to the frequency of the correct response, relative to the frequencies of other responses which might be made.

A series of problems can be constructed such that, for each problem, the possible responses are given, and the correct response occupies a particular position in terms of frequency relative to the incorrect responses. If $\mathrm{S}$ can identify this relative position, he will be able to select the correct response on subsequent problems with fewer errors.

Underwood \& Schulz (1960) demonstrated that Ss were able to judge accurately which of two bigrams (two-letter combinations) occurs more frequently in English, and that accuracy improved with increasing differences in frequency. Further evidence which might be relevant was reported by Schulz (1955), who had Ss first learn a serial list and then identify the ordinal positions of the items when these were presented randomly。 Results indicated that the middle positions were most difficult to identify. If this applies to a set of responses ordered in terms of frequency, then $\mathrm{S}$ should be better able to identify positions of high or low relative frequency, compared to positions of medium relative frequency. Therefore, in a series of problems whose solutions represent the same relative frequency position, $S$ should be better able to reduce errors if the correct item represents a position of high or low relative frequency than if the correct item occupies a position of medium relative frequency.
Overall performance was expected to be a function both of spew behavior and of the ability to identify relative frequency positions. The spew hypothesis predicts that problem difficulty will increase as the relative frequency of the correct response becomes lower. The "identification hypothesis" predicts that performance (over a series) will be better with correct responses of high or low relative frequency, compared to that with correct responses of medium relative frequency. Combining these predictions, as the correct response represents a lower relative frequency position, performance should deteriorate, then stabilize or show slight improvement.

\section{Method}

Materials. Each problem card contained five bigrams having the same initial letter. Based on the Underwood \& Schulz (1960) counts, bigrams with the same initial letter had been ranked, with rank 1 assigned to the most frequent bigrams in each set. The five bigrams in a single problem represented ranks $1,3,6,10$, and 15 for that set. It should be noted that, as an example, rank 15 indicates that 14 other bigrams have the same initial letter and higher frequencies. There were 10 problems, based on initial letters $a, e, h, m, n, o, r$, $s, u$, and $y$. For various ranks (over problems), the number of different second letters varied between six and nine, and particular letters appeared unsystematically in different rank positions in different problems. Latin square techniques were used to determine the spatial positions of bigrams on the cards and to construct ten different orders of the problems.

Design. The independent variable was the rank position (in terms of relative frequency) of the correct item on each problem. In a given condition, the same rank position was correct on all problems. There were five conditions, corresponding to whether rank $1,3,6,10$ or 15 was correct.

Subjects and procedure. As a course requirement, 10 introductory psychology students served as Ss in each condition. Each $\mathrm{S}$ was run individually and solved 10 problems, with the same rank position correct on all problems. Instructions indicated that the task was to select the correct item on each card in the smallest number of "guesses." The S could work at his own rate and was told that the items on a card varied in terms of their frequency of occurrence in English, and that the correct item had been designated on the basis of its frequency relative to the frequencies of the other items on the card. 


\section{Results}

Based on total number of errors over 10 problems, the means for the five conditions are presented in Table 1. Analysis of variance indicated that rank position of the correct response had a linear effect (F, 1/45, =19.79; $\mathrm{p}<.001$ ) and a quadratic effect (F, $1 / 45,=12.79 ; \mathrm{p}<.001$ ) on problem difficulty. The remaining deviations reached minimal significance, but these were relatively small $(F, 2 / 45,=5.09 ; \mathrm{p}<.05)$ 。 There was a general trend toward more errors as the correct response represented a lower relative frequency position, with a reversal beyond rank position 6 .

On the first problem, $S$ conceivably responded primarily on the basis of previously learned habits, and the spew hypothesis alone should apply. Errors on the first problem increased systematically with a decrease in the relative frequency position of the correct response (Table 1). Analysis of variance revealed a linear effect of rank position $(F, 1 / 45,=11.40 ; p<.005)$, which accounted for $87 \%$ of the between-groups variation.

\section{Discussion}

The results supported both the spew hypothesis and the identification hypothesis. Initial attempts at problem solution were predicted by the spew hypothesis, with responses of highest relative frequency given first. Over a series of problems, the response sequence may be altered by information concerning the position of the correct response in terms of relative frequency. The results indicate that $\mathrm{S}$ has difficulty using this information when the correct response occupies a
TABLE I

Mean Number of Errors

\begin{tabular}{lrrrrr} 
& \multicolumn{6}{c}{ Rank Position of Correct Respon se } \\
\hline & 1 & 3 & 6 & 10 & 15 \\
\hline All problems & 11.8 & 13.3 & 22.0 & 17.6 & 20.0 \\
First problem & 0.8 & 1.4 & 1.8 & 2.4 & 2.5 \\
\hline
\end{tabular}

position of medium relative frequency, but that positions of low relative frequency are easier to identify, and performance improves.

The present study suggests that different behavior will occur with a homogeneous set of problems, compared to behavior with a heterogeneous set, because of the (potential) use of additional information when solutions are similar. Safren (1962) found such a difference, in that anagram solving performance was superior when solutions were associated words, compared to performance with unrelated solutions. In the present study, Ss apparently discriminated the relative strengths of possible responses to change their problem solving behavior.

\section{References}

Safren, M. A. Associations, sets, and the solution of word problems. J. exp. Psychol., 1962, 64, 40-45.

Schulz, R. W. Generalization of serial position in rote serial learning. J. exp. Psychol., 1955, 49, 267-272.

Underwood, B. J., \& Schulz, R. W. Meaningfulness and verbal learning. Chicago: Lippincott, 1960.

Note

1. The assistance of Sharon Kestnbaum in collecting the data is appreciated. 